\title{
POLARIZED NEUTRON MATTER WITH SKYRME FORCES
}

\author{
Marek Kutschera \\ H. Niewodniczański Institute of Nuclear Physics \\ ul. Radzikowskiego 152, 31-342 Kraków, Poland \\ and \\ Włodzimierz Wójcik \\ Institute of Physics, Cracow University of Technology \\ ul. Podchorążych 1,30-084 Kraków, Poland
}

November 1993

\begin{abstract}
:
It is shown that Skyrme forces with a commonly used parameter $t_{2}<0$ lead to instability of polarized neutron matter. This instability has a form of the ferromagnetic spin ordering. There is, however, no ground state and the system displays a singular behaviour. Physically consistent description of polarized neutron matter requires that the exchange parameter $x_{2}$, which was neglected in spin-saturated systems, is in the range $-5 / 4<x_{2} \leq-1$. We find that Skyrme forces with $x_{2}$ in this range provide very good parameterization of realistic neutron matter calculations.
\end{abstract}

PACS numbers: 21.65.+f; 97.60.Jd

Simplicity of the Skyrme potential [1] makes it a useful tool in nuclear structure calculations $[2,3]$ and in studying properties of dense matter in neutron stars $[4,5]$. Here we are concerned with the latter case. 
The Skyrme potential used in astrophysical calculations is [5]

$$
\begin{gathered}
v(\mathbf{r})=t_{0}\left(1+x_{0} P_{\sigma}\right) \delta(\mathbf{r})+\frac{1}{2} t_{1}\left(1+x_{1} P_{\sigma}\right)\left[\mathbf{k}^{\prime 2} \delta(\mathbf{r})+\delta(\mathbf{r}) \mathbf{k}^{2}\right] \\
+t_{2}\left(1+x_{2} P_{\sigma}\right) \mathbf{k}^{\prime} \cdot \delta(\mathbf{r}) \mathbf{k}+v_{3}
\end{gathered}
$$

Here $\mathbf{r}=\mathbf{r}_{1}-\mathbf{r}_{2}$. The operator $\mathbf{k}=\frac{i}{2}\left(\nabla_{1}-\nabla_{2}\right)$ acts on the right of the delta-function and $\mathbf{k}^{\prime}$ is the same operator acting on the left of $\delta(\mathbf{r}) . P_{\sigma}$ is the spin exchange operator.

The 3-body term $v_{3}=t_{3} \delta\left(\mathbf{r}_{1}-\mathbf{r}_{2}\right) \delta\left(\mathbf{r}_{2}-\mathbf{r}_{3}\right)$ is equivalent to a two-body density dependent term [2] $(1 / 6) t_{3}\left(1+P_{\sigma}\right) n(\mathbf{R}) \delta(\mathbf{r})$, where $\mathbf{R}=\frac{1}{2}\left(\mathbf{r}_{1}+\mathbf{r}_{2}\right)$. In the following we shall use a modified form due to Ravenhall, Bennet and Pethick [4] who included in this expression an exchange term proportional to a new parameter $x_{3}$.

The energy density in a general case is

$$
\begin{gathered}
H(\mathbf{r})=\frac{\hbar^{2}}{2 m} \tau+\frac{1}{2} t_{0}\left[\left(1+\frac{1}{2} x_{0}\right) \rho^{2}-\left(x_{0}+\frac{1}{2}\right)\left(\rho_{N}^{2}+\rho_{P}^{2}\right)\right]+\frac{1}{4}\left[t_{1}\left(1+\frac{1}{2} x_{1}\right)+t_{2}\left(1+\frac{1}{2} x_{2}\right)\right] \rho \tau \\
+\frac{1}{4}\left[t_{2}\left(\frac{1}{2}+x_{2}\right)-t_{1}\left(\frac{1}{2}+x_{1}\right)\right]\left(\rho_{N} \tau_{N}+\rho_{P} \tau_{P}\right)-\frac{1}{16}\left[t_{2}\left(1+\frac{1}{2} x_{2}\right)-3 t_{1}\left(1+\frac{1}{2} x_{1}\right)\right](\nabla \rho)^{2} \\
-\frac{1}{16}\left[t_{2}\left(\frac{1}{2}+x_{2}\right)+3 t_{1}\left(\frac{1}{2}+x_{1}\right)\right]\left[\left(\nabla \rho_{N}\right)^{2}+\left(\nabla \rho_{P}\right)^{2}\right]+\frac{1}{4} t_{3}\left(\rho_{N} \rho_{P}+\frac{1}{4} x_{3}\left(\rho_{N}-\rho_{P}\right)^{2}\right) \rho .
\end{gathered}
$$

Here $\tau_{N}$ and $\tau_{P}$ are kinetic energy densities of, respectively, neutrons and protons, $\rho_{N}$ and $\rho_{P}$ are their number densities, $\tau=\tau_{N}+\tau_{P}$ and $\rho=\rho_{N}+\rho_{P}$.

The expression (2) contains 8 parameters $t_{0}, t_{1}, t_{2}, t_{3}, x_{0}, x_{1}, x_{2}$ and $x_{3}$ which are to be adjusted to fit properties of nuclei and nuclear matter. In most fits exchange parameters $x_{1}$ and $x_{2}$ are neglected, $x_{1}=x_{2}=0$.

Choice of the parameters is briefly described below. An important feature of various parameter sets $[1,2,3]$ is that often the parameter $t_{2}$ is negative, $t_{2}<0$, for $x_{2}=0$. In 
Table I we give two sets of Skyrme force parameters: The first one, SI, due to Vautherin and Brink [2] and the second one, SII, due to Beiner et al. [3]. There exist other sets in Ref.[3] with $t_{2}>0$ and very weak three-body force. In astrophysical calculations $[4,5]$, however, negative values of $t_{2}$ were commonly used.

In this note we point out that restricted Skyrme potential, i.e. with $x_{1}=x_{2}=0$ and with $t_{2}<0$ is inadequate to describe spin-polarized neutron matter. It leads to a singular behaviour of a polarized neutron matter. Any finite density solution is therefore metastable and there is no ground state. This is due to suppressing the exchange term in p-wave interaction by putting $x_{2}=0$. We show that the Hamiltonian is stabilized by including this term with a properly chosen exchange parameter $x_{2}$.

The instability becomes manifest if we consider fully polarized neutron matter with a uniform density. In this case the energy density is

$$
\epsilon_{f}=\frac{\hbar^{2}}{2 m} \tau_{u}+\frac{1}{2} t_{2} \tau_{u} \rho_{N}^{u}
$$

where $\tau_{u}=(3 / 5)\left(6 \pi^{2} \rho_{N}^{u}\right)^{2 / 3} \rho_{N}^{u}$ and $\rho_{N}^{u}=\rho_{N}$ is the density of ncutrons with spin up. This formula shows that for $t_{2}<0$ and $x_{2}=0$, as in both parameter sets in Table I, the energy has no lower bound and the system collapses to a singularity. The energy per particle for ferromagnetic neutron matter in this case is shown in Fig.1 (dashed line with $x_{2}=0$ ). Thus the Hamiltonian we use is not well defined. It should be improved by including a new term making the system stable.

The physical origin of this instability is rather obvious. The Skyrme potential is a contact potential with momentum-dependent terms representing s-wave and p-wave interactions. All these terms contribute in spin-saturated systems. However, for ferromagnetic neutron matter the Pauli principle suppresses completely all s-wave terms, and the only term surviving is the p-wave contribution proportional to $t_{2}$. Since this term has stronger density dependence than kinetic energy term it always wins at high densities and leads to a singularity when $t_{2}<0$. 
In order to make the Hamiltonian well defined one should include the exchange term in the p-wave term which was neglected by putting $x_{2}=0$. That is the only way to stabilize ferromagnetic neutron matter with Skyrme potential (1) since p-wave interaction is the only term which contributes in the ferromagnetic state.

Including $x_{2}$ the energy density of the ferromagnetic neutron matter becomes

$$
\epsilon_{f}=\frac{\hbar^{2}}{2 m} \tau_{u}+\frac{1}{2} t_{2}\left(1+\dot{x}_{2}\right) \tau_{u} \rho_{N}^{u}
$$

This formula shows that $x_{2} \leq-1$ is required in order to make the Hamiltonian stable, for negative values of $t_{2}$. Of course, no $x_{2}$ is needed for $t_{2}>0$.

Let us now briefly describe how the parameter $x_{2}$ can be determined. The Skyrme force parameters are adjusted in first place to fit properties of symmetric nuclear systems where $\rho_{N}=\rho_{P}=\rho / 2$. For such systems the Hamiltonian simplifies to

$$
\begin{gathered}
H(\mathbf{r})=\frac{\hbar^{2}}{2 m} \tau+\frac{3}{8} t_{0} \rho^{2}+\frac{1}{16}\left[3 t_{1}+5 t_{2}\left(1+\frac{4}{5} x_{2}\right)\right] \rho \tau \\
+\frac{1}{64}\left[9 t_{1}-5 t_{2}\left(1+\frac{4}{5} x_{2}\right)\right](\nabla \rho)^{2}+\frac{1}{16} t_{3} \rho^{3} .
\end{gathered}
$$

One can notice that this formula contains only one exchange parameter $x_{2}$. All other exchange parameters, $x_{0}, x_{1}$ and $x_{3}$ cancel out in symmetric nuclear systems and hence cannot be determined from properties of these systems. Let us also note that the values of parameters $t_{2}$ and $x_{2}$ cannot be determined separately since these appear in two terms above in the same product

$$
\bar{t}_{2} \equiv t_{2}\left(1+\frac{4}{5} x_{2}\right)
$$

By fitting properties of symmetric nuclear systems one can determine the values of $t_{0}, t_{1}, t_{3}$ and $\bar{t}_{2}$. In calculations shown in Figs.1 and 2 we adopt the values obtained by Vautherin and Brink [2] who fit saturation properties of nuclear matter and energies of 
${ }^{16} \mathrm{O}$ and ${ }^{208} \mathrm{~Pb}$ nuclei (SI force in Table I). The latter nucleus is slightly asymmetric, however its asymmetry parameter is small.

Saturation properties of nuclear matter give the following relations:

$$
\begin{gathered}
t_{0} \rho_{0}=\frac{4}{9} K+\frac{40}{3} W-\frac{16}{5} T_{F}, \\
\frac{3}{16} t_{3} \rho_{0}^{2}=K+15 W-\frac{9}{5} T_{F}, \\
\frac{1}{16}\left[3 t_{1}+t_{2}\left(5+4 x_{2}\right)\right] k_{F}^{2} \rho_{0}=2 T_{F}-15 W-\frac{5}{6} K,
\end{gathered}
$$

where the saturation density $\rho_{0}=0.155 \mathrm{fm}^{-3}$ and the saturation energy $W=-16 \mathrm{MeV}$ are input parameters in the fit of Ref.[2]. Corresponding value of the incompressibility modulus is $K=370 \mathrm{MeV}$, somewhat above the empirical walue of $220 . \mathrm{IEV}$. It is a general feature of Skyrme forces that the incompressibility is a little too high.

Requirement of stability of polarized neutron matter and the cmpirical result that $\bar{t}_{2}<0$ allow us to constrain the range of $x_{2}$. The parameter $x_{2}$ should satisfy inequalities

$$
\bar{t}_{2} \equiv t_{2}\left(1+\frac{4}{5} x_{2}\right)<0 \quad \text { and } \quad t_{2}\left(1+x_{2}\right) \geq 0 .
$$

These two conditions can be fulfilled only if $t_{2}<0$ and $-5 / 4<x_{2} \leq-1$. We find thus a remarkably narrow range for physically allowed values of $x_{2}$ for $\bar{t}_{2}<0$.

Remaining exchange parameters, $x_{0}, x_{1}$ and $x_{3}$, enter the formulae for asymmetric nuclear systems. We shall focus on uniform density systems. The symmetry energy is

$$
E_{s y m}=\frac{1}{3} T_{F}-\frac{1}{4} t_{0}\left(x_{0}+\frac{1}{2}\right) \rho_{0}-\frac{1}{16} t_{3}\left(1-x_{3}\right) \rho_{0}^{2}+\frac{1}{24}\left(-3 t_{1} x_{1}+t_{2}\left(4+5 x_{2}\right)\right) k_{F}^{2} \rho_{0},
$$

where $k_{F}=\left(1.5 \pi^{2} \rho_{0}\right)^{1 / 3}$ and $T_{F}=k_{F}^{2} / 2 m$.

The energy density of a uniform neutron matter reads 


$$
\begin{aligned}
H(\mathbf{r})= & \frac{\hbar^{2}}{2 m} \tau_{N}+\frac{1}{4} t_{0}\left(1-x_{0}\right) \rho_{N}^{2}+\frac{1}{8}\left[t_{1}\left(1-x_{1}\right)+3 t_{2}\left(1+x_{2}\right)\right] \rho_{N} \tau_{N} \\
& +\frac{3}{32}\left[t_{1}\left(1-x_{1}\right)-t_{2}\left(1+x_{2}\right)\right]\left(\nabla \rho_{N}\right)^{2}+\frac{1}{16} t_{3} x_{3} \rho_{N}^{3} .
\end{aligned}
$$

Here $\tau_{N}=(3 / 5) k_{N}^{2} \rho_{N}$ with $k_{N}=\left(3 \pi^{2} \rho_{N}\right)^{1 / 3}$. Since the parameter $x_{1}$ is irrelevant to both ferromagnetic neutron matter and symmetric nuclear matter in the following we shall put $x_{1}=0$ for simplicity.

In Ref.[2] it is assumed that $x_{1}=x_{2}=0$ and $x_{3}=1$. From eq.(11) the value of $x_{0}$ is then obtained, assuming empirical value $E_{s y m}=30 \mathrm{MeV}$. Resulting equation of state of neutron matter is, however, too stiff as compared with realistic neutron matter calculations. To obtain better description of neutron matter a lower value $x_{3}=0.52$ was adopted in Ref.[4]. This leads to different value for $x_{0}$.

In this paper we adjust the exchange parameters $x_{2}$ and $x_{3}$ to obtain an overall good reproduction of neutron matter calculations of Wiringa, Fiks and Fabrocini [6]. As can be seen in Fig.2, we reproduce the energies rather well. Particularly interesting is the case of $U V 14+T N I$ interaction, which is very nicely reproduced with $x_{2}=-1.08$ and $x_{3}=0$, solid line in Fig.2. For comparison we show the dashed curve corresponding to $x_{2}=0$ and $x_{3}=0.3\left(x_{0}=0.42\right)$. Clearly, the curve with no three-body force, $x_{3}=0$, and $x_{2}=-1.08$ fits the calculations of Ref.[6] much better. The $U V 14+U V I I$ interaction has stronger three-body force contribution and to reproduce the energy of neutron matter the exchange parameter $x_{3}=0.24$ is used.

In Table II we give the values of the exchange parameters $x_{0}, x_{2}$ and $x_{3}$ adjusted to reproduce the $U V 14+T N I$ and $U V 14+U V I I$ results from Ref.[6] (solid curves in Fig.2). - They are obtained for the SI Skyrme force in Table I. Also, the exchange parameters corresponding to the SII force from Table I, are given.

The energy of the ferromagnetic neutron matter is shown in Fig.1 for $x_{2}=-1.08$ as a dashed line. It increases monotonically with density and lies above energy of normal 
neutron matter with $U V 14+T N I$ interactions. This means that normal neutron matter is the ground state of the system at any density. The reason of this behaviour is the fact that there is no three-body interaction in neutron matter, $x_{3}=0$, in this case.

For other interactions from Ref.[6] $x_{3}>0$ and at high enough densities the ferromagnetic neutron matter becomes the ground state of the system. This occurs always when there is a three-body contribution present in normal neutron matter energy since the energy of ferromagnetic matter has weaker density dependence.

In conclusion, consistent description of polarized neutron matter with Skyrme forces requires that for negative values of the parameter $t_{2}$ corresponding exchange parameter $x_{2}$ is in a narrow range $-5 / 4<x_{2} \leq-1$. Skyrme forces with properly chosen exchange parameters $x_{2}$ and $x_{3}$ provide very good representation of realistic neutron matter calculations.

This work was partially supported by KBN grants 202049101 and 200549101 .

\section{REFERENCES}

[1] T. H. R. Skyrme, Nucl. Phys. 9, 615 (1959).

[2] D. Vautherin and D. M. Brink, Phys. Rev. C 5, 626 (1972).

[3] M. Beiner, H. Flocard, Nguyen Van Giai and P. Quentin, Nucl. Phys. A238, 29 (1975).

[4] D. G. Ravenhall, C. D. Bennett and C. J. Pethick, Phys. Rev. Lett. 28, 978 (1972).

[5] J. M. Lattimer, Ann. Rev. Nucl. Part. Sci. 31, 337 (1981).

[6] R. B. Wiringa, V. Fiks and A. Fabrocini, Phys. Rev. C 38, 1010 (1988). 


\section{TABLE I}

Skyrme force patulueters: $S I$ from Ref.[2] and $S I I$ from Ref.[3]

\begin{tabular}{|c|c|c|c|c|}
\hline Force & $t_{0}\left[M e V f m^{3}\right]$ & $t_{1}\left[M e V f m^{5}\right]$ & $\bar{t}_{2}\left[\mathrm{MeV} \mathrm{fm} \mathrm{m}^{5}\right]$ & $t_{3}\left[M e V f m^{6}\right]$ \\
\hline $\mathrm{SI}$ & -1057.3 & 235.9 & -100.0 & 14463.5 \\
\hline SII & -1128.8 & 395.0 & -95.0 & 14000.0 \\
\hline
\end{tabular}

\section{TABLE II}

Exchange parameters of Skyrme forces $S I$ and $S I I$ in Table I

\begin{tabular}{|c|c|c|c|c|}
\hline Force & Equation of state & $x_{0}$ & $x_{2}$ & $x_{3}$ \\
\hline \multirow[t]{2}{*}{ SI } & UV14+TNI & 0.19 & -1.08 & 0.00 \\
\hline & UV14+UVII & $0.1 i$ & -1.02 & 0.24 \\
\hline \multirow[t]{2}{*}{ SII } & $\mathrm{UV} 14+\mathrm{TNI}$ & 0.28 & -1.00 & 0.00 \\
\hline & UV14+UVII & 0.16 & -1.06 & 0.07 \\
\hline
\end{tabular}




\section{FIGURE CAPTIONS}

Fig.1

Energy per particle of normal neutron matter (solid line) and ferromagnetic neutron matter (dashed lines) as a function of neutron density. Open squares represent calculations of Ref.[6] for the $U V 14+T N I$ interaction. Solid line is the $S I$ Skyrme force fit with parameters given in Table I and exchange parameters from Table II. Upper dashed line is for stable ferromagnetic matter whereas the lower one corresponds to singular case with $x_{2}=0$.

Fig.2

The same as in Fig.1 for normal neutron matter results from Ref.[6]. Solid lines are $S I$ Skyrme force fits with parameters in Tables I and II. For comparison results with $x_{2}=0$ are shown as dashed lines. Corresponding values of remaining exchange parameters for the $U V 14+T N I$ (squares) and $U V 14+U V I I$ (triangles) interactions are, respectively, $x_{0}=0.42, x_{3}=0.30$ and $x_{0}=0.32, x_{3}=0.48$. 
Fig. 1

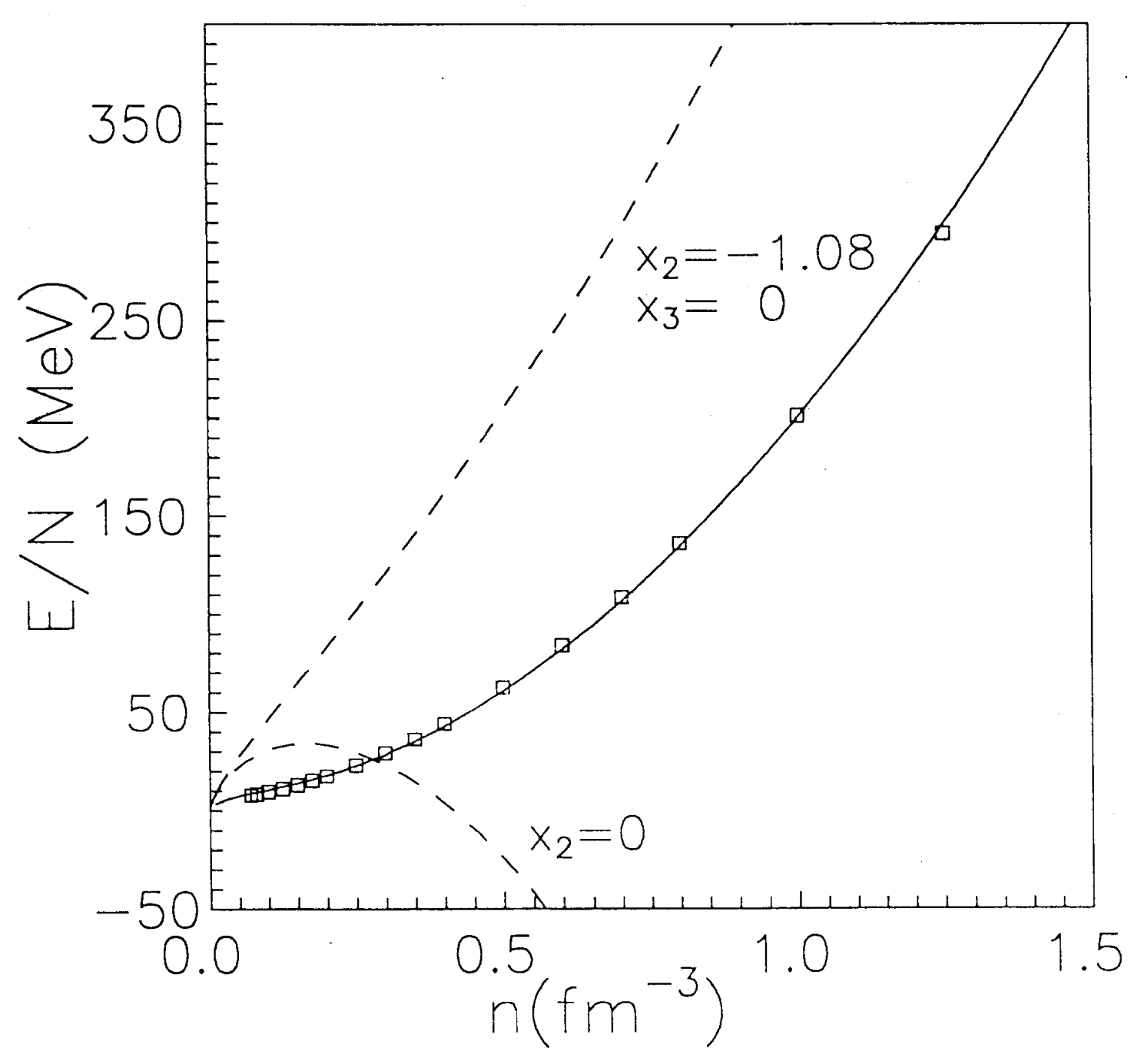


Fig. 2

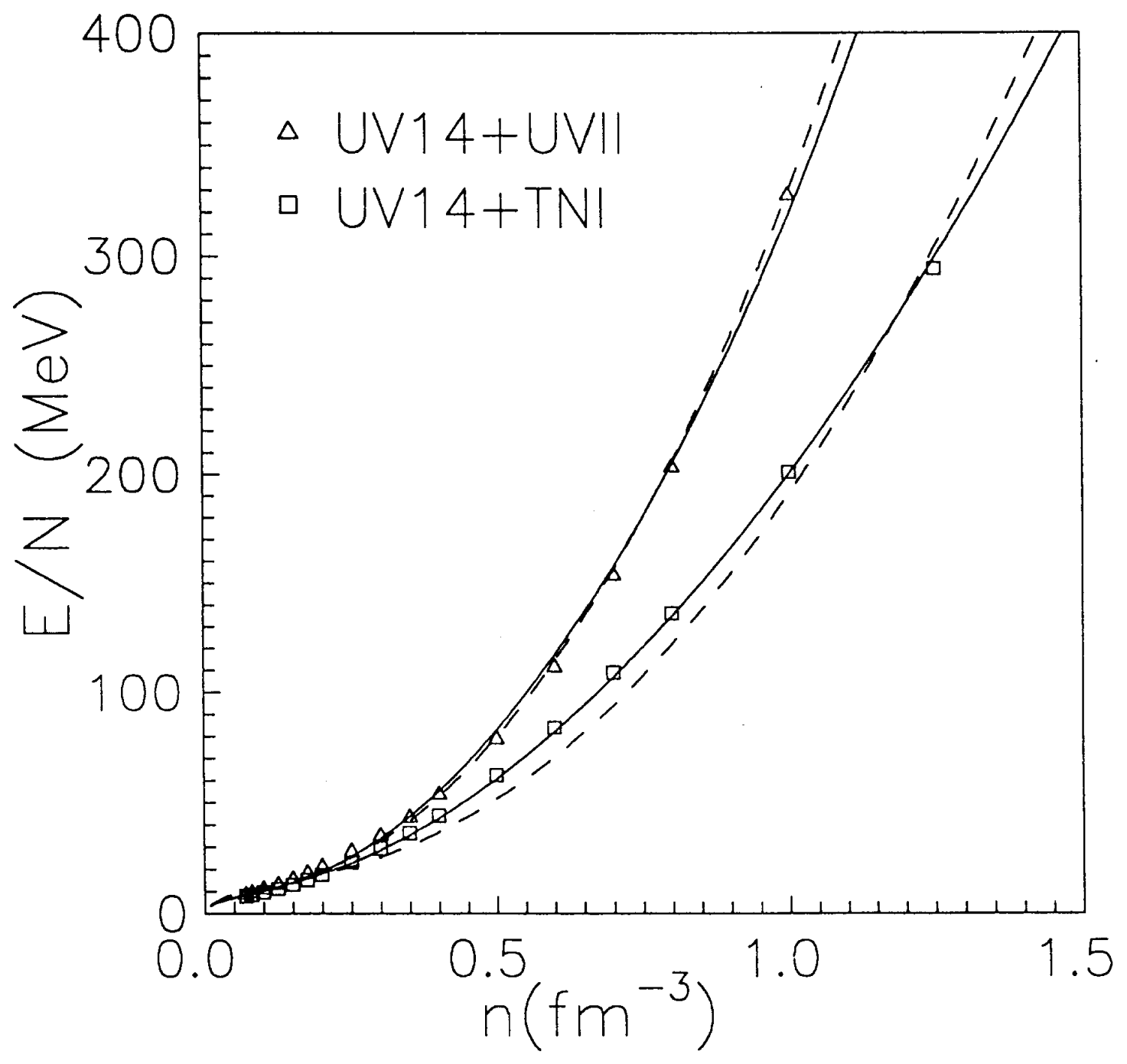


